\title{
Proposal and Verification of Simultaneous Measurement Method for Three Thermoelectric Properties with Film-Type Thermocouple Probe
}

\author{
Takumi Yamazaki $^{1}$, Ai Ueno ${ }^{2}$, Hosei Nagano² \\ ${ }^{1}$ Department of Aerospace Engineering, Nagoya University, Nagoya, Japan \\ ${ }^{2}$ Department of Mechanical Science and Engineering, Nagoya University, Nagoya, Japan \\ Email: tyamazaki@prop2.nuae.nagoya-u.ac.jp
}

How to cite this paper: Yamazaki, T., Ueno, A. and Nagano, H. (2017) Proposal and Verification of Simultaneous Measurement Method for Three Thermoelectric Properties with Film-Type Thermocouple Probe. Journal of Electronics Cooling and Thermal Control, 7, 23-32. https://doi.org/10.4236/jectc.2017.72003

Received: May 4, 2017

Accepted: May 30, 2017

Published: June 2, 2017

Copyright $\odot 2017$ by authors and Scientific Research Publishing Inc. This work is licensed under the Creative Commons Attribution International License (CC BY 4.0).

http://creativecommons.org/licenses/by/4.0/

\begin{abstract}
A new simultaneous measurement method for the measurement of the three thermoelectric properties with a film-type thermocouple probe was proposed. Seebeck coefficient was measured using the steady-state condition of the differential method. The electrical resistivity was measured using the four-probe method and the thermal diffusivity is measured using the periodic heating method. The effectiveness of the proposed method was verified using constantan as a reference material. After describing the effectiveness of the method, the measurement of three thermoelectric properties of $\mathrm{Bi}_{0.3} \mathrm{Sb}_{1.7} \mathrm{Te}_{3}$, which is a thermoelectric material, was performed.
\end{abstract}

\section{Keywords}

Film-Type Thermocouple Sensor, Simultaneous Measurement, Thermoelectric Properties

\section{Introduction}

Thermoelectric power generation is attracting attention as an unutilized energy utilization method. On the other hand, it has not been widely used because of its low energy conversion efficiency, toxic or rare components in its material and so on. Therefore, new thermoelectric materials are being actively developed to solve these problems. Technologies to evaluate these materials with high precision have become increasingly important. The thermoelectric material is evaluated using the dimensionless figure of merit $Z T$. $Z T$ is expressed as 


$$
Z T=\frac{S^{2}}{\rho \kappa} T
$$

where $S$ is the Seebeck coefficient, $\rho$ is electrical resistivity, $\kappa$ is thermal conductivity, and $T$ is the absolute temperature. In evaluating the performance of thermoelectric materials, it is necessary to measure three physical property values $S$, $\rho$ and $\kappa$. Conventionally, temperature dependency of these properties was measured separately in order to calculate $Z T$. In other words, it takes a great deal of time and effort to evaluate the performance of materials. Materials with nanostructures, which have been developed in recent years, are anisotropic, and there are reports that $Z T$ greatly differ depending on the literature in spite of the same material ([1]). This is because the property values of the material vary depending on the measurement position. In other words, measurement under the same condition is required for accurate performance evaluation. As a means to solve these problems, simultaneous physical property values measurement can be cited.

Currently, there are several simultaneous measurement methods for two thermoelectric properties such as the Seebeck coefficient and electric resistivity, or the Seebeck coefficient and thermal conductivity. However, the number of previous studies of simultaneous measurement which can measure three thermoelectric properties are limited. Chaussy et al. ([2]) developed an apparatus for simultaneous measurement of three thermoelectric properties at a temperature range of 1.2 - $350 \mathrm{~K}$. The steady state method was adopted for the thermal conductivity measurement. The measurement of the thermal conductivity at 1.2 $100 \mathrm{~K}$ shows good results with an error of $10 \%$, however, at $100-350 \mathrm{~K}$, the error is maximum as $25 \%$. Although Kallaher et al. ([3]) developed a simultaneous measurement device at 300 - $673 \mathrm{~K}$, electrical resistivity could not be directly calculated from the measured electrical resistance because the contact area of the electrode to the sample is large and the shape cannot be corrected. Vasilevskiy et al. ([4]) and Kolb et al. ([5]) devised a device that can cross-check $Z T$ based on the Harman method ([6]) while measuring three thermoelectrical property values in the same setting. However, Harman method, which can measure $Z T$ directly, is not strict measurement because it does not take heat loss due to Joule heat into account. Furthermore, the former did not measure the accurate temperature difference and thermoelectromotive force of the sample in the Seebeck coefficient measurement because the thermocouples were placed in the electrode with temperature gradient. In the latter measurement, the length between the voltage measurement lead wires was not reflected in deriving the electrical resistivity. Martin et al. ([7]) achieved temperature dependence measurement at 12 - $300 \mathrm{~K}$, however the influence of heat loss due to thermal radiation cannot be ignored above this temperature.

As described above, in the temperature range above the room temperature, there is still no effective method of simultaneous measurement of thermoelectric properties. In this paper, a new simultaneous measurement method for three thermoelectric properties with film-type thermocouple probe has been proposed. 
At the beginning, the principle of the measurement and the original instrument is explained. In order to verify the proposed measurement method, constantan is used as a reference material. In addition, the result of the temperature dependence measurements of constantan is reported. Finally, the measurement result of $\mathrm{Bi}_{0.3} \mathrm{Sb}_{1.7} \mathrm{Te}_{3}$ is shown and discussed.

\section{Proposed Measurement Method and Instrument}

\subsection{Measurement Principle}

The principle of the simultaneous measurement for three thermoelectric properties will be explained using Figure 1.

1) The Seebeck coefficient (Figure 1(a))

The Seebeck coefficient measurement is conducted under the steady-state condition. By heating the one edge of the sample, a temperature gradient is generated along the longitude direction of the sample. From the temperature difference $\Delta T$ and the thermoelectromotiveforce $\Delta V_{t h}$ between two measuring points, the relative Seebeck coefficient between the sample and the wire are obtained as the Equation (2).

$$
S_{\text {sample-wire }}=-\Delta V_{t h} / \Delta T
$$

The absolute Seebeck coefficient of the sample can be obtained by adding the absolute Seebeck coefficient $S_{\text {wire }}$ to the measured relative Seebeck coefficient $S_{\text {sample-wire }}$

$$
S_{\text {sample }}=S_{\text {sample-wire }}+S_{\text {wire }}
$$

2) Electrical resistivity (Figure 1(b))

Four-probe method is used as a measuring method for electrical resistivity. As the current $I$ is applied to the installed leads to the sample, the voltage drop $\Delta V$ between any two points on the sample is measured. The electrical resistivity is obtained with the sample thickness $t$ as the Equation (4).

$$
\rho=\frac{\pi}{\ln 2} t \frac{\Delta V}{I} f_{1} f_{2}
$$

where $t$ is the sample thickness and $f_{1}$ and $f_{2}$ are shape correction factors ([8]) which are expressed as

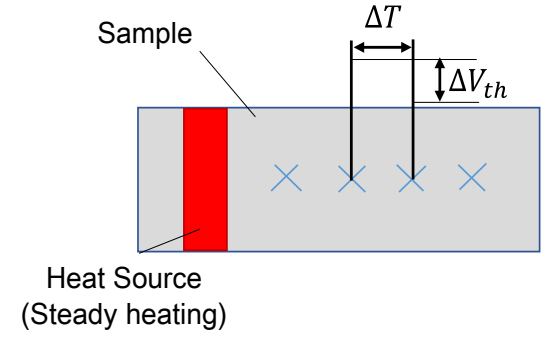

(a)

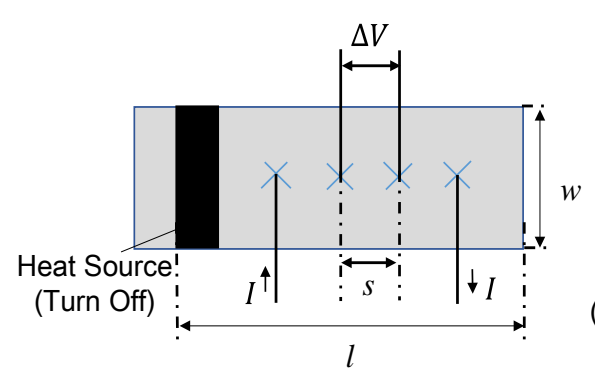

(b)

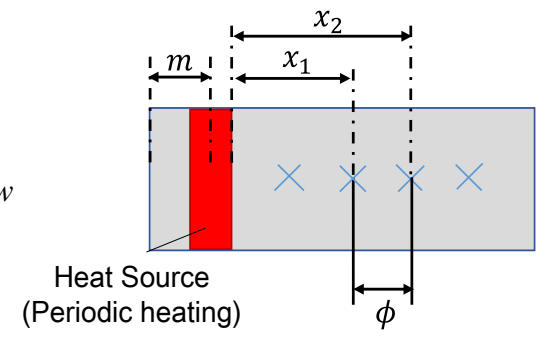

(c)

Figure 1. Conceptual schemes of measurement principles. (a) the Seebeck coefficient (b) electrical resistivity (c) thermal diffusivity. 


$$
\begin{gathered}
f_{1}=\frac{\ln 2}{\ln \{\sinh (t / s) / \sinh (t / 2 s)\}} \\
f_{2}=f_{2 R}\left(\frac{l}{w}, \frac{w}{l}\right)
\end{gathered}
$$

where $s, l$ and $w$ are the probe pitch, the sample length and the sample width, respectively.

In order to eliminate the effect of thermoelectromotive force due to Joule heat, the polarity of the current is switched and the average value of the obtained voltage drops is used as $\Delta V$ in Equation (4).

3) Thermal diffusivity (Figure 1 (c))

In the thermal diffusivity measurement, periodic heating is used. By heating periodically one end of the sample, the AC temperature is propagated along the longitude direction of the sample. Considering the one dimensional heat conduction equation with respect to the sample length direction $x$, the phase delay $\phi$ of the AC temperature is expressed as

$$
\phi=\sqrt{\frac{\pi f}{a}} x
$$

where $a$ is thermal diffusivity of the sample and $f$ is the frequency of the AC temperature. In Equation (7), when $x$ is a constant $x_{1}$ and $x_{2}$ and $\phi$ is a function of only $\sqrt{f}$, both sides are differentiated by $\sqrt{f}$ and sorted out, Equation (8) is obtained.

$$
a=\pi\left(x_{1}-x_{2}\right)^{2} /\left(\frac{\mathrm{d} \phi}{\mathrm{d} \sqrt{f}}\right)^{2}
$$

In other words, by measuring the phase delay at the two points $x_{1}$ and $x_{2}$ on the sample when the heating frequency is changed, $\mathrm{d} \phi / \mathrm{d} \sqrt{f}$ is obtained and the thermal diffusivity a can be calculated. In this method, because thermal diffusivity is calculated from the phase difference of the AC temperature, it is characterized by being hardly affected by heat loss. Equation (7) is solved under the assumption that the sample is an infinite flat plate and no thickness. Therefore, there exists the range of the heating frequency $\sqrt{f}$ at the Equation (7) holds ([9] [10]), and the measurement must be conducted within the range the following Equation (9).

$$
\frac{a}{\pi}\left(\frac{7 \pi}{8 m}\right)^{2}<f \leq \frac{a}{\pi}\left(\frac{1}{4 t}\right)^{2}
$$

In Equation (9), $m$ is the distance between the center of the heater and the one sample edge as the Figure 1(c) shows.

\subsection{Instruments}

1) Film-type thermocouple probe

The photograph of film-type thermocouple probe proposed in this research is shown in Figure 2. and its specifications are shown in Table 1. The film type thermocouple probe has two pairs of chromel-alumel thermocouples and two 


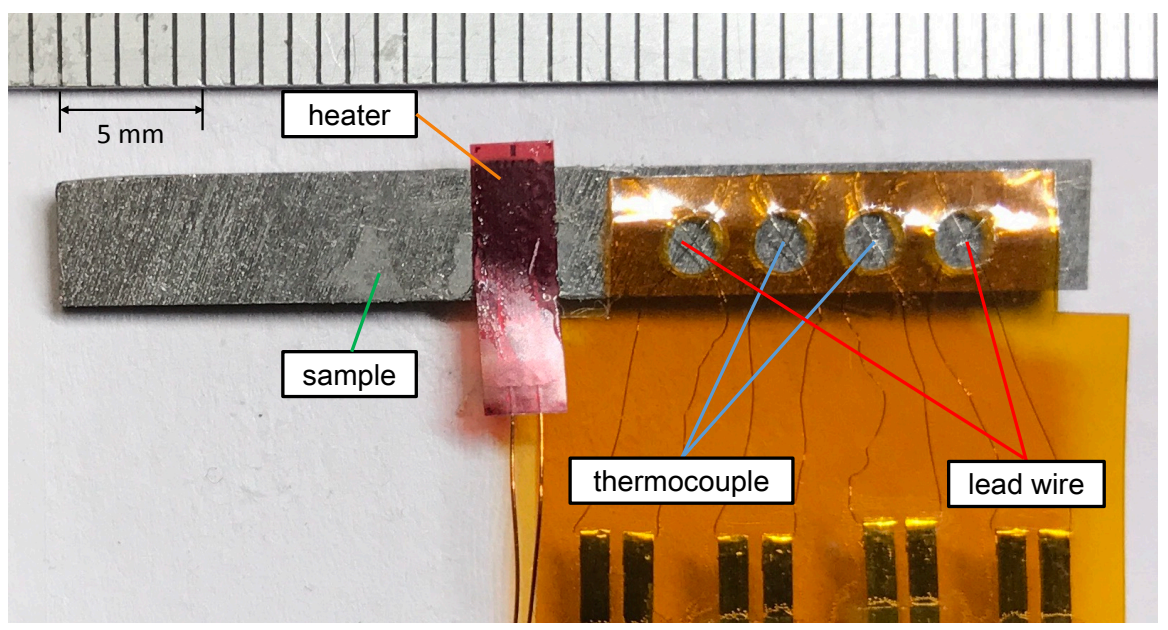

Figure 2. Film-type thermocouple probe.

Table 1. Specification of the film-type thermocouple probe.

\begin{tabular}{ccc}
\hline \multicolumn{2}{c}{ Components } & Material \\
\hline Base & Polyimide & $15 \times 2 \times t 0.05 \mathrm{~mm}$ \\
Thermocouple & Chromel, Alumel & $50 \phi \mathrm{m}$ \\
Lead wire & Constantan & $50 \phi \mathrm{m}$ \\
Sensor's pitch & - & $3 \mathrm{~mm}$ \\
Heater & Cu-Ni alloy & $6.3 \times 2.8 \mathrm{~mm}(120 \Omega)$ \\
\hline
\end{tabular}

pairs of lead wires at both ends. The former are used for the measurements of the temperature and the voltage difference. The latter are used in order to apply the current to the sample. In addition, a strain gauge is used as a heater for heating the sample, and it is installed so that it can perform one-dimensional heating in the in-plane direction of the sample.

2) Simultaneous measurement device

Figure 3 shows a conceptual diagram of the simultaneous measurement device. The measuring device consists of a measuring part, DC power supplies, a function generator, a data logger, a nanovoltmeter, a lock-in amplifier, a switch box, and relays. All measuring devices are connected to the PC by GPIB, and they are controlled and recorded by LabVIEW. In the Seebeck coefficient measurement, a heater is heated using a DC power supply and make the temperature gradient along the in-plane direction of the sample. And that time, the data logger is used to measure the temperature difference occurring between the two points of the sample fixed with thermocouples. Measurement of thermoelectromotive force caused by temperature difference is measured with the nanovoltmeter with the thermocouple's chromel wire as the lead wire. For electric resistivity measurement, the DC power supply is used for current application to the constantan lead wire. The voltage drop between two thermocouples is measured with the nanovoltmeter with the thermocouple's chromel wire as the lead wire. In thermal diffusivity measurement, the periodic signal to the heater uses the output voltage of the function generator. The phase difference of the AC tem- 


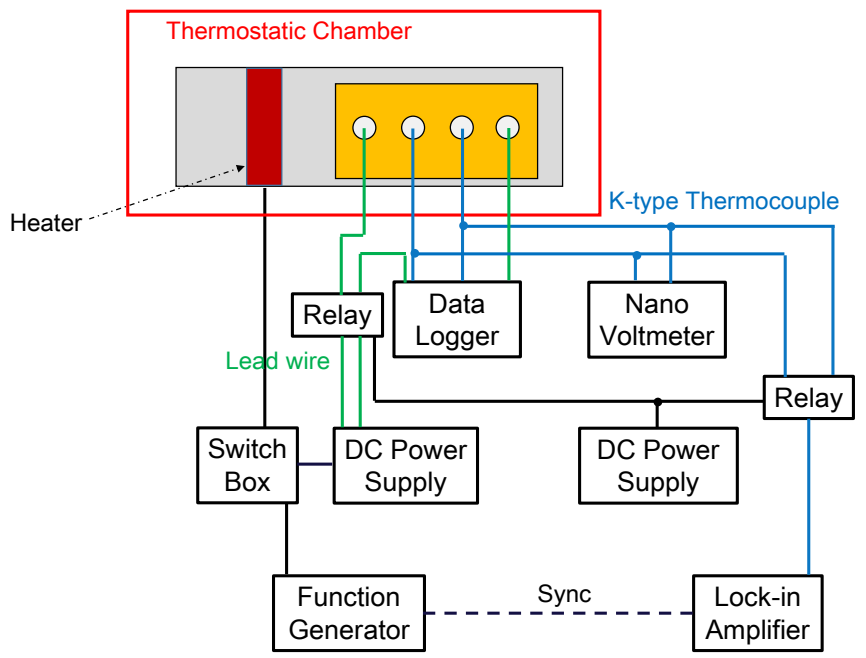

Figure 3. The conceptual diagram of the simultaneous measurement device.

perature measured between two thermocouples is measured using the lock-in amplifier. These three thermoelectric property measurements can be done automatically by switching the wiring using relays. Based on the measurement principle shown in the previous section, it is possible to measure thermoelectric properties of bulk material with the same setting. By placing the measuring part into a thermostatic chamber, the atmospheric temperature can be controlled to enable temperature dependency measurements.

\section{The Verification of the Proposed Measurement Instrument}

In order to verify the effectiveness of this method, measurement of three thermoelectric properties at $293 \mathrm{~K}$ was carried out using constantan (Cu: 50\% - 55\%, Ni: $45 \%-50 \%)$ as a reference sample. The sample size was $5 \times 36 \times t 0.3 \mathrm{~mm}$. The reason for choosing constantan as the reference sample here is that it is possible to verify the Seebeck coefficient measurement by using the thermoelectromotive force table ([11]) of the E-type thermocouple as a reference by using chromel as the element wire. Figure 4 shows the measurement results of (a) the Seebeck coefficient, (b) electrical resistivity and (c) thermal diffusivity, respectively. The values of each property were calculated from the slopes of each plot using the formulas (2), (4) and (8).

Table 2 shows the measurement values of the relative Seebeck coefficient, electrical resistivity and thermal diffusivity calculated with the reference values and their differences. From this result, all the difference value fell within $\pm 10 \%$. In addition, this measurement was able to show the repeatability. Therefore, the effectiveness of this measuring device is verified.

\section{Application to Temperature Dependence Measurement}

Temperature dependence of thermoelectric properties was measured using this 


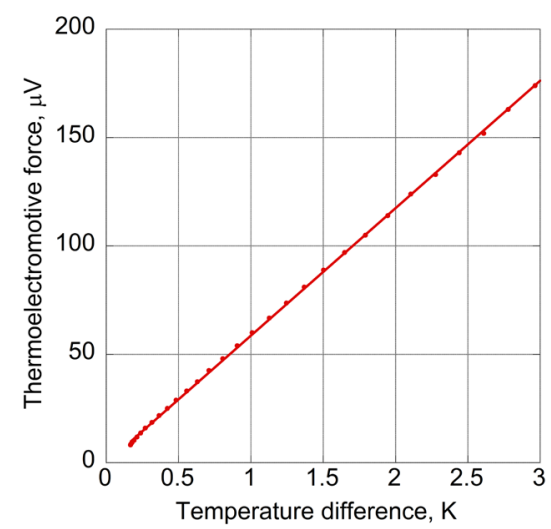

(a)

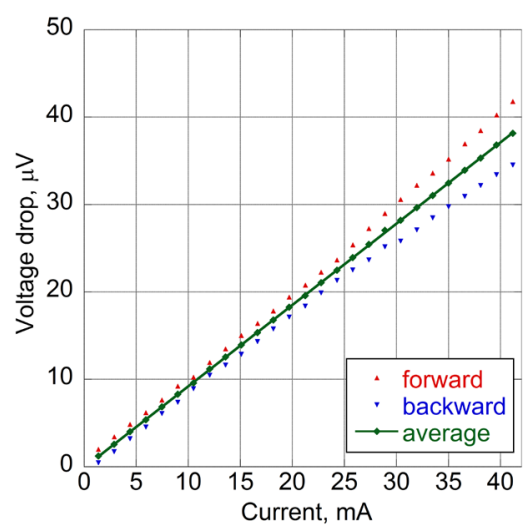

(b)

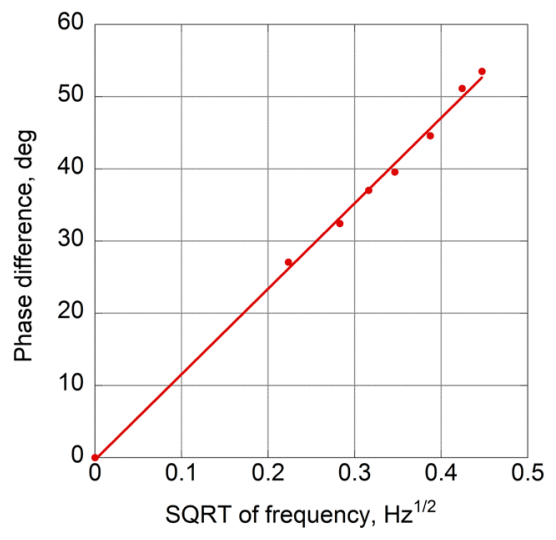

(c)

Figure 4. All typical plot on each measurement (constantan). (a) The thermoelectromotive force and the temperature difference (b) The voltage drop and the applied current. (c) The phase difference and the squareroot of the frequency.

Table 2. Measurement results and the reference values of thermoelectric properties of constantan and the difference between measurement results and reference value (293 K).

\begin{tabular}{ccccccc}
\hline & \multicolumn{2}{c}{ Relative Seebeck coefficient } & \multicolumn{2}{c}{ Electrical resistivity } & \multicolumn{2}{c}{ Thermal diffusivity } \\
\cline { 2 - 7 } & 1st run & 2nd run & 1st run & 2nd run & 1st run & 2nd run \\
\hline Measurement result & $59.4 \mu \mathrm{V} / \mathrm{K}$ & $58.8 \mu \mathrm{V} / \mathrm{K}$ & $0.468 \mu \Omega \cdot \mathrm{m}$ & $0.466 \mu \Omega \cdot \mathrm{m}$ & $6.63 \mathrm{~mm}^{2} / \mathrm{s}$ & $5.99 \mathrm{~mm}^{2} / \mathrm{s}$ \\
Reference value & $60.5 \mu \mathrm{V} / \mathrm{K}([11])$ & $0.486 \mu \Omega \cdot \mathrm{m}([12])$ & & $6.34 \mathrm{~mm}^{2} / \mathrm{s}([13])$ \\
Difference & $-1.8 \%$ & $-2.8 \%$ & $-3.7 \%$ & $-4.1 \%$ & $-4.5 \%$ & $5.5 \%$ \\
\hline
\end{tabular}

measurement method. The measurement part was placed in a thermostatic chamber and the measurement was carried out while changing the temperature inside the thermostatic chamber at intervals of $10 \mathrm{~K}$ in the range of 283 to $343 \mathrm{~K}$. The measurement results are shown in Figure 5. From these graphs, it was found that the difference of each property measurement between the measurement result and the reference value are within the range of $-2.0 \%$ to $2.0 \%$ for the Seebeck coefficient, $-4.7 \%$ to $0.4 \%$ for electrical resistivity and $0.7 \%$ to $7.1 \%$ for thermal diffusivity.

\section{Measurement of Thermoelectric Properties with $\mathrm{Bi}_{0.3} \mathrm{Sb}_{1.7} \mathrm{Te}_{3}$ and Discussion}

\subsection{Measurement Result ( $\left(\mathrm{Bi}_{0.3} \mathrm{Sb}_{1.7} \mathrm{Te}_{3}\right)$}

Three thermoelectric properties of $\mathrm{Bi}_{0.3} \mathrm{Sb}_{1.7} \mathrm{Te}_{3}$ which was known a representative thermoelectric material were measured. The sample size was $4.5 \times 35 \times t 1$ $\mathrm{mm}$. This $\mathrm{Bi}_{0.3} \mathrm{Sb}_{1.7} \mathrm{Te}_{3}$ was sinter prepared by a hot press method. The measurement was carried out by placing the apparatus in a thermostatic chamber and the absolute Seebeck coefficient of the sample was calculated by substituting the absolute Seebeck coefficient of chromel ([14]) used as a wire in Equation (3). Figure 6 shows the measurement results of (a) the Seebeck coefficient, (b) electrical resistivity and (c) thermal diffusivity. Table 3 shows their results together with the reference values and their differences. The differences were $0.3 \%, 11.7 \%$ 


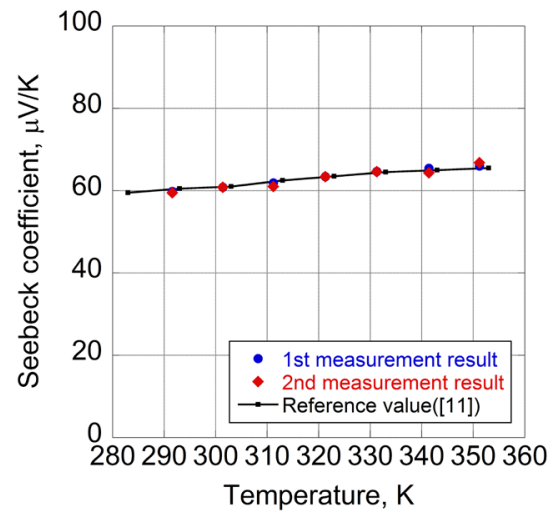

(a)

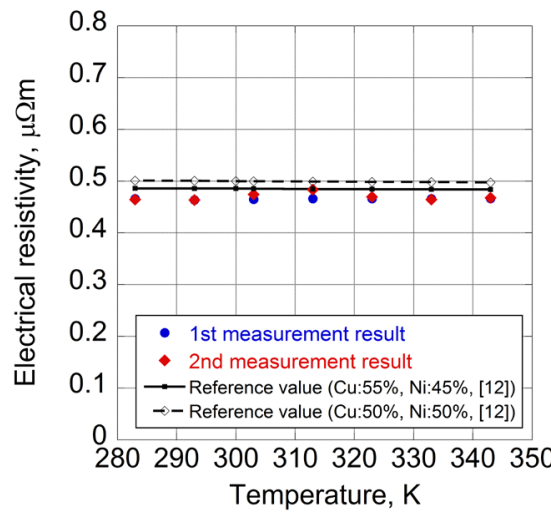

(b)

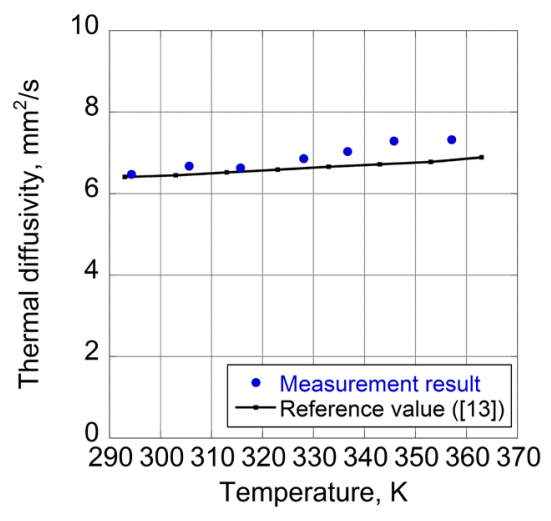

(c)

Figure 5. The measurement result of temperature dependency for each property (constantan). (a) The Seebeck coefficient; (b) Electrical resistivity; (c) Thermal diffusivity.

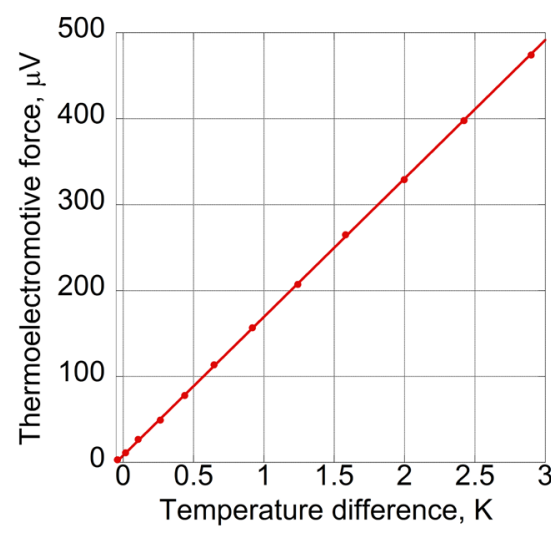

(a)

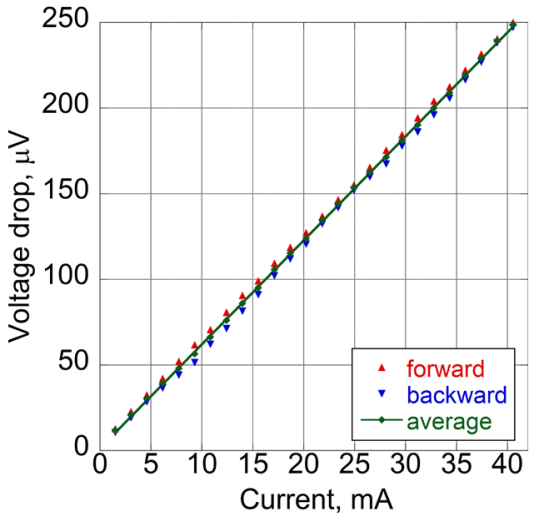

(b)

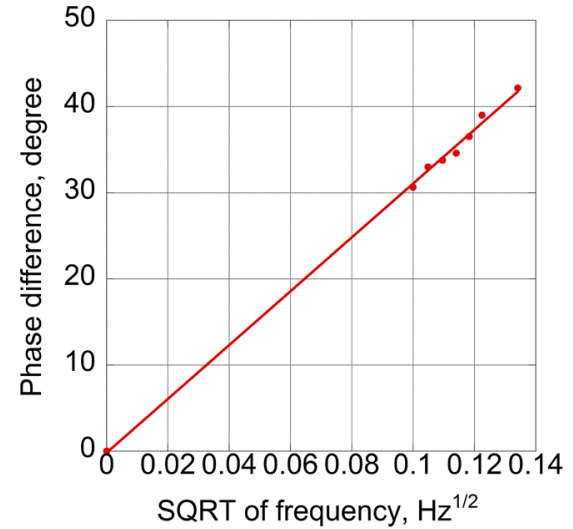

(c)

Figure 6. All typical plot on each measurement $\left(\mathrm{Bi}_{0.3} \mathrm{Sb}_{1.7} \mathrm{Te}_{3}\right)$. (a) The thermoelectromotive force and the temperature difference (b) The voltage drop and the applied current. (c) The phase difference and the square root of the frequency.

Table 3. Measurement results and the reference values of thermoelectric properties of $\mathrm{Bi}_{0.3} \mathrm{Sb}_{1.7} \mathrm{Te}_{3}$ and the difference between measurement results and reference value.

\begin{tabular}{cccc}
\hline & Seebeck coefficient & Electrical resistivity & Thermal diffusivity \\
\hline Measurement result & $183.3 \mu \mathrm{V} / \mathrm{K}$ & $9.44 \mu \Omega \cdot \mathrm{m}$ & $1.01 \mathrm{~mm}^{2} / \mathrm{s}$ \\
Reference value & $182.8 \mu \mathrm{V} / \mathrm{K}([15])$ & $10.7 \mu \Omega \cdot \mathrm{m}([15])$ & $0.88 \mathrm{~mm}^{2} / \mathrm{s}([16])$ \\
Difference & $0.3 \%$ & $11.7 \%$ & $14.8 \%$ \\
\hline
\end{tabular}

and $14.8 \%$ for the Seebeck coefficient, electrical resistivity and thermal diffusivity, respectively.

\subsection{Discussion}

In the measurement of electrical resistivity and thermal diffusivity of $\mathrm{Bi}_{0.3} \mathrm{Sb}_{1.7} \mathrm{Te}_{3}$, the cause of the large difference between the measurement result and the reference value will be considered.

1) Electrical resistivity

When a current $I$ is applied to the thermoelectric material, heat absorption 
and heat generation occur by the Peltier effect at the junction between electrode and the material as an effect peculiar to the thermoelectric material. The heat quantity $Q$ is expressed as

$$
Q=I T S_{\text {sample-wire }}
$$

This heat generates a temperature gradient in the sample, and a thermoelectromotive force is generated. The thermoelectromotive force due to the Peltier heat cannot be canceled even if the polarity of the current is changed unlike thermoelectromotive force caused by Joule heat. This thermoelectromotive force is added to the drop voltage due to the resistance and it is measured to be larger than the actual resistance. As a solution, it is possible to measure in a short time after applying the current by utilizing the fact that the relaxation time of heat is significantly longer than that of the career. ([17])

2) Thermal diffusivity

In the case of constantan, the difference between the measured value and the reference value was $4.5 \%$. In the case of $\mathrm{Bi}_{0.3} \mathrm{Sb}_{1.7} \mathrm{Te}_{3}$, however, it was a large value of $14.8 \%$. This may be attributed to the difference in the value of thermal diffusivity between the two materials. While the thermal diffusivity of constantan is $6.34 \mathrm{~mm}^{2} / \mathrm{s}$ ([13]), that of $\mathrm{Bi}_{0.3} \mathrm{Sb}_{1.7} \mathrm{Te}_{3}$ is $0.88 \mathrm{~mm}^{2} / \mathrm{s}([16])$ and is about $1 / 8$ size of constantan. In the case of $\mathrm{Bi}_{0.3} \mathrm{Sb}_{1.7} \mathrm{Te}_{3}$, the AC temperature amplitude decayed at the thermocouple contact position. Therefore, by reducing the distance between the thermocouple contact position and the heater, it can be considered that the measurement is conducted more accurately. Thermoelectric materials are low-thermal conductivity materials like $\mathrm{Bi}_{0.3} \mathrm{Sb}_{1.7} \mathrm{Te}_{3}$ and it is considered that it is necessary to measure the temperature in the vicinity of the heater. However, since the sample has a finite thickness at a location close to the heater, one-dimensional thermal conductivity does not hold true. Therefore, by solving the two-dimensional heat conduction equation, it may be possible to estimate the boundary between the position where one-dimensional thermal conductivity is established and the position till which it does not hold true, and it is also necessary to investigate the optimum position to contact the thermocouple.

\section{Conclusion}

A new measurement method for thermoelectric properties was proposed and the verification of this method using a film-type thermocouple probe was carried out using constantan. The difference between the physical property values and the reference value was within $\pm 10 \%$, indicating the soundness of the device. Further, three thermoelectric property values of $\mathrm{Bi}_{0.3} \mathrm{Sb}_{1.7} \mathrm{Te}_{3}$ were measured at a temperature of $323 \mathrm{~K}$ (thermal diffusivity measurement is $300 \mathrm{~K}$ ), and the difference between the measured value and the reference value was as follows: the result was $0.3 \%, 11.7 \%$ and $14.8 \%$ for the Seebeck coefficient, electric resistivity and thermal diffusivity, respectively.

\section{Acknowledgements}

Part of this research funding was subsidized by strategic fundamental technology advancement support project. The author wish to acknowledge Kiyoshi Ogawa 
and Ryouzo Hiramatsu of Ozawa Science Co., Ltd. and Dr. Woosuck Shin of AIST cooperated in promoting this research.

\section{References}

[1] Chen, N., et al. (2005) Macroscopic Thermoelectric in Homogeneities in $\left(\mathrm{AgSbTe}_{2}\right)_{x}(\mathrm{PbTe})_{1-x}$. Applied Physics Letters, 87, 171903.

[2] Chaussy, J., Guessous, A. and Mazuer, J. (1981) Simultaneous Measurements of Thermopower, Thermal Conductivity, and Electrical Resistivity between 1.2 and 350 K. Review of Scientific Instruments, 52, 1721. https://doi.org/10.1063/1.1136520

[3] Kallaher, R.L., Latham, C.A. and Sharifi, F. (2013) An Apparatus for Concurrent Measurement of Thermoelectric Material Parameters. Review of Scientific Instruments, 84, 013907.

[4] Valsilevskiy, D., Simard, J.-M., Masut, R.A. and Turenne, S. (2015) System for Simultaneous Harman-Based Measurement of All Thermoelectric Properties, from 240 to $720 \mathrm{~K}$, by Use of a Novel Calibration Procedure. Journal of Electronic Materials, 44, 1733.

[5] Kolb, H., Dasgupta, T., Zabrocki, K., Mueller, E. and de Boor, J. (2015) Simultaneous Measurement of All Thermoelectric Properties of Bulk Materials in the Temperature Range 300-600 K. Review of Scientific Instruments, 86, 073901.

[6] Harman, T.C. (1958) Special Techniques for Measurement of Thermoelectric Properties. Journal of Applied Physics, 29, 1373. https://doi.org/10.1063/1.1723445

[7] Martin, J., et al. (2016) Apparatus for the Measurement of Electrical Resistivity, Seebeck Coefficient, and Thermal Conductivity of Thermoelectric Materials between $300 \mathrm{~K}$ and $12 \mathrm{~K}$. Review of Scientific Instruments, 87-015105.

[8] Smits, F.M. (1958) Measurement of Sheet Resistivities with the Four-Point Probe. The Bell System Technical Journal, 37, 711. https://doi.org/10.1002/j.1538-7305.1958.tb03883.x

[9] Takahashi, H., et al. (1998) Proceedings of the 9 th Japan Symposium on Thermophysical Properties, B222.

[10] Gu, Y. and Hatta, I. (1991) Effect of Sample Edge in ac Calorimetric Method for Measuring Thermal Diffusivity of Thin Films with High Thermal Diffusivity. Japanese Journal of Applied Physics, 30, 1137.

[11] IEC 584-1 (1977) Thermocouples Part 1: Reference Tables.

[12] Ho, C.Y., et al. (1983) Electrical Resistivity of Ten Binary Alloy Systems. Journal of Physical and Chemical Reference Data, 12, 183. https://doi.org/10.1063/1.555684

[13] Sundqvist, B. (1992) Thermal Diffusivity and Thermal Conductivity of Chromel, Alumel, and Constantan in the Range 100 - 450 K. Journal of Applied Physics, 72, 539. https://doi.org/10.1063/1.351885

[14] Bentley, R.E. (1998) Handbook of Temperature Measurement Vol. 3: Theory and Practice of Thermoelectric Thermometry. Springer, Berlin, 31.

[15] Yamamoto, A., et al. (2009) Evaluation of Homogeneity of Bi2Te3-Based Materials Prepared by Hot-Press Method. Proceedings of the 6th Annual Meeting of the Thermoelectrics Society of Japan, S2-2.

[16] Li, J., et al. (2013) BiSbTe-Based Nanocomposites with High ZT: The Effect of SiC Nanodispersion on Thermoelectric Properties. Advanced Functional Materials, 23, 4317-4323. https://doi.org/10.1002/adfm.201300146

[17] Isoda, Y. (2016) Tips for an Evaluation Method for Thermoelectric Conversion Materials. Oyo Buturi, 85, 1043. 
Submit or recommend next manuscript to SCIRP and we will provide best service for you:

Accepting pre-submission inquiries through Email, Facebook, LinkedIn, Twitter, etc. A wide selection of journals (inclusive of 9 subjects, more than 200 journals)

Providing 24-hour high-quality service

User-friendly online submission system

Fair and swift peer-review system

Efficient typesetting and proofreading procedure

Display of the result of downloads and visits, as well as the number of cited articles Maximum dissemination of your research work

Submit your manuscript at: http://papersubmission.scirp.org/

Or contact jectc@scirp.org 